\title{
Neuroimaging Strategies Addressing Challenges in Using fMRI for the Children with Cerebral Palsy
}

\author{
Juniper J. Lee-Park ${ }^{*}$, Harshawardhan Deshpande², Jonathan Lisinski2, Stephen LaConte1,2,3, \\ Sharon Ramey1,4,5,6,7, Stephanie DeLuca1,4,5,6,7
}

${ }^{1}$ Virginia Tech Carilion School of Medicine, Roanoke, VA, USA

${ }^{2}$ Virginia Polytechnic Institute and State University, Blacksburg, VA, USA

${ }^{3}$ Emergency Medicine, Virginia Tech Carilion School of Medicine, Roanoke, VA, USA

${ }^{4}$ Neuromotor Research Clinic, Virginia Tech Carilion Research Institute, Roanoke, VA, USA

${ }^{5}$ Department of Pediatrics, Virginia Tech Carilion School of Medicine, Roanoke, VA, USA

${ }^{6}$ Psychology, Virginia Polytechnic Institute and State University, Blacksburg, VA, USA

${ }^{7}$ Department of Rehabilitation, Health, and Wellness, Jefferson College of Health Sciences, Roanoke, VA, USA

Email: *ljunip2@vt.edu

How to cite this paper: Lee-Park, J.J., Deshpande, H., Lisinski, J., LaConte, S., Ramey, S. and DeLuca, S. (2018) Neuroimaging Strategies Addressing Challenges in Using fMRI for the Children with Cerebral Palsy. Journal of Behavioral and Brain Science, 8, 306-318.

https://doi.org/10.4236/jbbs.2018.85019

Received: April 4, 2018

Accepted: May 14, 2018

Published: May 17, 2018

Copyright (ㅇ 2018 by authors and Scientific Research Publishing Inc. This work is licensed under the Creative Commons Attribution International License (CC BY 4.0).

http://creativecommons.org/licenses/by/4.0/

\begin{abstract}
Aim: This study sought to develop a process and methodology that could be a useful clinical and research tool for successfully completing functional magnetic resonance imaging (fMRI) scanning in children with Cerebral Palsy. Method: Six children with CP (mean age of 8.83 years; five with spastic hemiplegia, one with spastic quadriplegia) and three children with typical development (mean age of 9.33 years) completed an fMRI scanning protocol that used real-time motion feedback as a means of minimizing head and trunk motion. Anatomical, resting-state, and motor-task scans were sequentially obtained from each subject. Precentral "hand-knob" regions were identified on the anatomical scan and served as seed regions to reveal the functional connectivity of each subject's brain as associated with hand movement. Results: Real-time motion feedback aided children in successful completion of resting state scans. Functional connectivity and brain activity mapping were obtained based on anatomical landmarks, and laterality indices were developed based on the obtained functional-connectivity map to specify a dominant side of brain activity that was matched to a clinical profile, despite anatomical variations that occur with Cerebral Palsy. Interpretation: Real-time motion feedback and the development of laterality indices can improve the clinical and research utility of fMRI scanning. What this paper adds: 1)
\end{abstract}


Presents a real-time imaging protocol for fMRI to help children complete scanning; 2) Presents an fMRI methodology to obtain laterality indices in the presence of abnormal anatomy; 3) Provides findings of LI that match clinical diagnosis.

\section{Keywords}

fMRI, Cerebral Palsy, Real-Time Motion Feedback, Functional-Connectivity, Resting State

\section{Introduction}

Cerebral palsy $(\mathrm{CP})$ is a spectrum of non-progressive disorders of the developing brain that affects posture and motor control [1]. In 2004, Taub, Ramey, DeLuca, and Echols demonstrated that pediatric constraint-induced movement therapy ( $\mathrm{p}$-CIMT) was efficacious in helping young children with hemiparetic CP make clinically significant gains in function. Since then intensive therapies have consistently demonstrated efficacy in the treatment of hemiparesis [2] [3] [4] [5] in helping them overcome some of the associated functional limitations. However, there is still little documentation about the potential impact of rehabilitation treatment on brain function. Although, most clinicians and researchers assume that intensive treatments cause neuroplasticity but this is only an assumption with little corresponding evidence.

This knowledge gap exists because there are significant impediments in successfully using neuroimaging with children. Functional magnetic resonance imaging (fMRI) is viewed as one of the most powerful tools to investigate neural plasticity, and has been used in many studies with adults and older children [6]. Unfortunately, for children with $\mathrm{CP}$, using fMRI technology is challenging. Two of the most frequently cited challenges include:

1) children with $\mathrm{CP}$ struggle to maintain head stability during scanning, especially during task-based performance necessary for motor scan, causing excessive motion artifact for data analysis, and

2) children with $\mathrm{CP}$ have additional anatomical variations, complicating the whole-brain mapping necessary for functional analysis [5] [6] [7] [8] [9].

fMRI scanning does hold great potential for both research and clinical purposes, because it allows a researcher to study brain activities when a subject is at rest or performing a given task. Therefore, a novel scanning protocol to address these two challenges, in other words, to minimize motion artifacts and facilitate functional mapping, might increase the usefulness of the data for children with varied disabilities, including CP. Furthermore, this would allow better examination of neural plasticity, including examination of how a rehabilitation treatment can alter the functional network of the brain.

Therefore, the goal of this study is to obtain such data that are minimally affected by the motion artifacts, yet yielding clinically accurate analysis. We sought 
to accomplish this goal by conducting a seed-voxel analysis on the resting state brain network, because, on one hand, resting scan data is relatively unaffected by the motion artifacts compared to the motor scan and on the other hand, it does not require a whole brain analysis as it is more focused on a particular "seed", or a region of interest (ROI). In order to obtain a quality resting scan data, we also adopted real-time (RT) motion feedback system to assist children in limiting head and neck motions during scanning [6] [7].

\section{Method}

\subsection{Participants}

Six participants with CP (mean age 8.83 y; five with spastic hemiplegia, one with spastic quadriplegia) were recruited for the study. Inclusion criteria were school aged children with known diagnoses of hemiplegic cerebral palsy, who were to receive pediatric constraint-induced movement therapy (p-CIMT) at the Virginia Tech Research Institute. Exclusion criteria for further data analyses were incompletion of p-CIMT, refusal for post-treatment fMRI, and lack of cooperation during scan leading to incomplete scanning session. Anatomical abnormality or head motion, if quantified as motion index $<1$, were not exclusion criteria. Three healthy children without known brain lesion participated (TDC; mean $9.33 \mathrm{y}$ ), and ten fMRI data sets from adults ( $>20 \mathrm{y}$ ) with no known neurological diagnoses were obtained from a data repository were used to validate identification of ROIs. The participants and legal guardians signed the consent form. The protocol was described and approved by the Institutional Review Board (IRB) of the Virginia Polytechnic Institute and State University.

\subsection{Standard Procedures}

All MRI scanning was performed on a Siemens 3T Allegra with physiological monitoring of vital signs. Collected anatomical $\mathrm{T} 1$ scan data were pre-processed by slice timing correction, conventional motion correction, spatial smoothing (6 mm Full Width at Half Maximum, FWHM) and band-pass filtering $(0.01 \mathrm{~Hz}$ to $0.08 \mathrm{~Hz}$ ). Each scanning session began with the 5-minute T1-weighted structural scan, followed by one 5 -minute resting state fMRI scan, and concluded with one 6-minute motor task fMRI scan. The T1 weighted anatomical images are acquired with a 3D MPRAGE sequence at $1 \mathrm{~mm}$ isotropic resolution and scanning parameters: $\mathrm{TI}=900 \mathrm{~ms}, \mathrm{TR}=2.6 \mathrm{~s}, \mathrm{TE}=3.02 \mathrm{~ms}, \mathrm{FA}=8^{\circ}, \mathrm{BW}=130 \mathrm{~Hz} /$ pixel for an acquisition time of 5 minutes.

\subsection{Using Resting Scan Data}

Functional MRI (fMRI) data were acquired using a $\mathrm{T} 2{ }^{\star}$ weighted single-shot EPI sequence. The whole-brain functional volumes are acquired in $303.4-\mathrm{mm}$ axial slices (20\% slice gap), each with $3.44 \mathrm{~mm} \times 3.44 \mathrm{~mm}$ in-plane resolution. Additional fMRI imaging parameters include: $\mathrm{TR}=2 \mathrm{~s}, \mathrm{TE}=30 \mathrm{~ms}, \mathrm{FA}=90^{\circ}, \mathrm{BW}=$ $2442 \mathrm{~Hz} /$ pixel. During both functional scans, including resting scan and motor 
scan, head motion was recorded during scanning from three translational and three rotational planes every repetition time $(\mathrm{TR}=2 \mathrm{sec})$. At each TR $(t)$, the amount of head motion was used to calculate a composite motion index $(M(t))$ to reject unacceptable amount of head motion for further data analysis (conventionally defined as $M(t)>1 \mathrm{~mm}[10][11]$

$$
\begin{gathered}
M(t)=\Delta M+w M(t-\Delta t) \\
\Delta M=\left(\sum_{n=1}^{t}\left(p_{n}(t)-p_{n}(t-\Delta t)\right)^{2}\right)^{1 / 2}
\end{gathered}
$$

Here, $w$ is a weight factor, and the $p$ values represent aforementioned six (three translational and three rotational) motion parameters at a time. In contrast to Laterality Index (LI) later used for functionality analysis, $M(t)$ is a quantification for head motion that is not related to brain functionality [12]. In order to perform data analysis that is as little affected by the motion artifact as possible, $M(t)$ was calculated and recorded while real-time motion feedback system was being used. In this system, $M(t)$ was translated into a visual representation of four-way arrow display that changed color from blue to red once a composite motion index (MI) exceeded an acceptable threshold [13] [14] [15] [16] (Figure 1).

Increased number of red arrows was programed to indicate increasing degrees of motion. Subjects were instructed to remain still during the scan with a goal of keeping the set of arrows shown on a screen inside the scanner blue indicating acceptable motion levels $(M(t)<1 \mathrm{~mm})$. Each subject was provided immediate feedback about their head motion via this representation projected into the

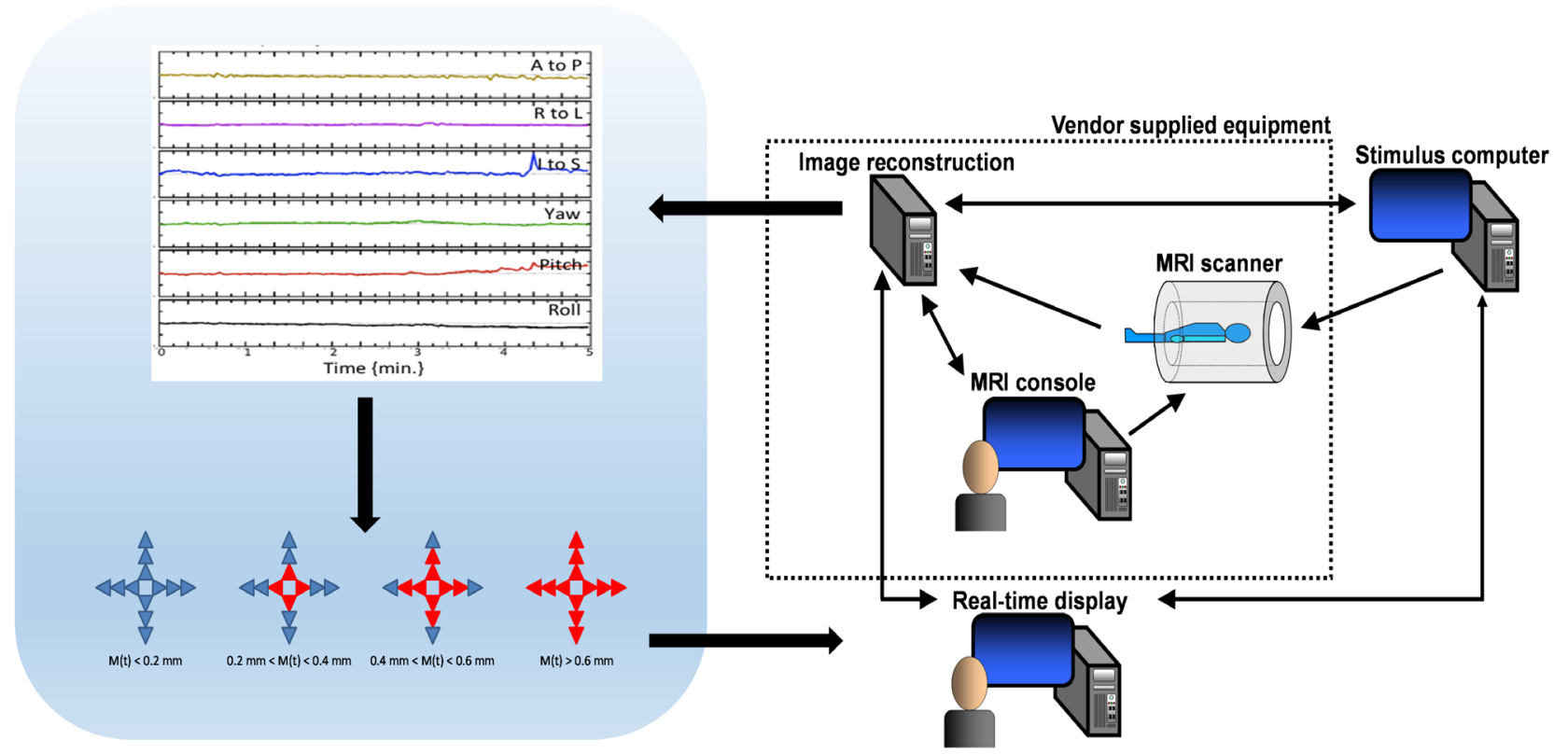

Figure 1. Real-time fMRI system. The dotted box represents subsystems which include the MRI scanner, console, and image reconstruction. For real-time display of motion parameters, there was an additional computer running AFNI in the control room that transmits motion parameters to control the stimulus display. Obtaining motion parameters in real-time has been effectively used to adapt pulse sequence parameters. 
scanner. This feedback method was given only during the resting scan, as its purpose was to optimize resting scan data. If a subject produced a head motion, exceeding $M(t)$ greater than $1 \mathrm{~mm}$, he or she was allowed to undergo more than one run. All valid runs with salvageable, or usable, data $\left(\mathrm{n}^{\mathrm{RUN}}\right.$; elapse time between the start time and time when an excessive head motion occurred $>1$ min) were included and averaged within each group.

\subsection{Region of Interest and Seed-Voxel Analysis}

The precentral "hand-knob" areas [17] [18] [19], which are known to be correlated to the hand and arm movement, were marked on the pre-processed $\mathrm{T} 1$ anatomical data as a pair of $6 \mathrm{~mm}$-radius sphere in each hemisphere. These were then overlapped with the resting scan data to identify the regions of interest (ROIs) on the resting state brain. Resulted ROIs served as seed-voxels for seed-voxel analysis, which revealed the relationship between the whole brain network and ROIs in the resting state. Identifying ROIs as well as alignment between anatomical and functional data sets were done by Edge detection function of Analysis of Functional Neuro Images (AFNI) neuroimaging analytical software (Scientific and Statistical Computing Core, National Institute of Mental Health, Bethesda, MD, US).

\subsection{Using Motor Task Scan Data}

The stimulus paradigms were created from the hemodynamic response of the brain and expressed as a brain activity map during a given motor task, finger tapping assignment. Subjects were instructed to follow the visual instructions by pressing a button on a fiber optic button box. A short practice session was given based on the subject's need and understanding of the instruction. Unlike other conventional studies, the purpose of motor task scan in our study was to validate anatomical hand-knob areas as functionally relevant ROI, rather than to simply visualize the active areas within the brain during a given task.

\subsection{Functional Connectivity Analysis}

From aforementioned ROIs, seed-voxel analysis was performed to yield inter-hemispheric laterality as a result. AFNI InstaCorr feature ("Instantaneous Correlation") was used for this purpose. Laterality index (LI), which signifies the predominant side of the brain activity during the given motor task, was used as a qualified measure for inter-hemispheric laterality. Conventionally LI equation is expressed as $\mathrm{f}(\mathrm{QLH}-\mathrm{QRH}) /(\mathrm{QLH}+\mathrm{QRH})$ where $\mathrm{QLH}$ and $\mathrm{QRH}$ are fMRI measurements for the $\mathrm{LH}$ and $\mathrm{RH}$ contributions. We used a modified version of this equation to create a LI for CP children, written as LI (Unaffected) $=(\mathrm{U}-$ $\mathrm{A}) /(\mathrm{U}+\mathrm{A})$ where $\mathrm{U}$ is a number of voxels from the unaffected side and $\mathrm{A}$ is from the affected side.

\subsection{Statistical Analysis}

A correlation was used to study the association between the amounts of head 
and neck motion and the time-course the scanning protocols. Analysis of variance was obtained to test the impact of using real-time motion feedback between two subject groups to understand difference in head and neck motion amounts between children with and without CP.

\section{Results}

\subsection{Real-Time Motion Feedback for Optimization of Resting Data}

When the feedback was given (Figure 2(a)), both groups were able to keep their heads still within the acceptable level of motion for further data analysis $(M(t)$ $<1 \mathrm{~mm}$; Equation (2)). Comparing the average motion indices within the groups, TDC subjects (blue) moved their heads more than the CP group (red), as the scanning sessions approached towards the end (time lapse $(t)=298-300$ $\mathrm{sec}$ ). When the feedback was stopped (Figure $2(\mathrm{~b})$ ), both subject groups resulted in greater head motions. Again looking at the average motion indices without feedback, CP subjects were significantly more motion-prone compared to their typically developing counterparts.

\subsection{Seed Voxel Analysis for Optimization of Motor Data}

For the functional scan, seed-voxel analysis was used based on the resting data obtained as above. Region of interest (ROI) for this seed-voxel analysis, also known as hand-knob areas, were easily identifiable in twenty adult hemispheres from healthy adults. Once ROIs were identified on T1 anatomical data, they were aligned with motor data to ensure their functional correlation with motor data. The overlapping region within the ROI on the resting data was calculated

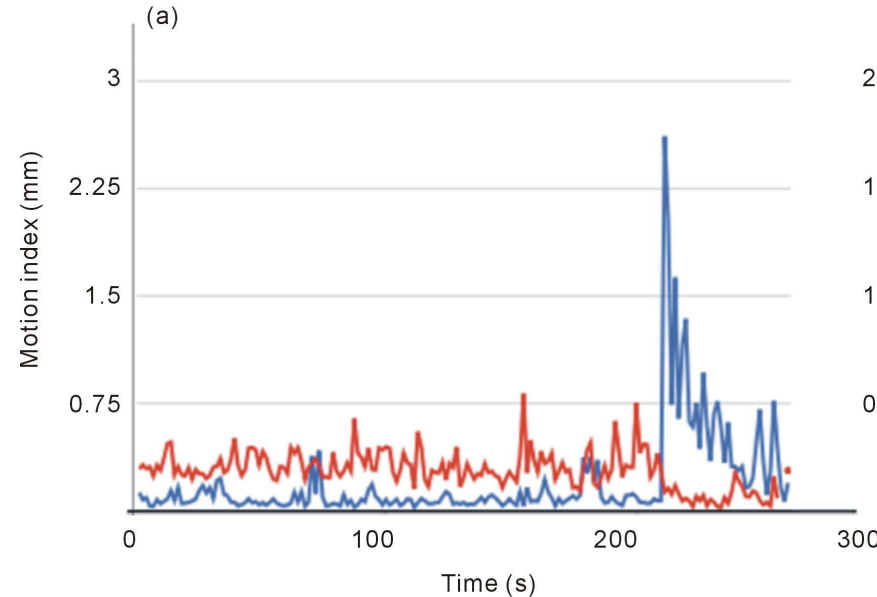

Typically developing (healthy) children $\left(n^{\text {subject }}=3, n^{\text {RUN }}=3\right)$

Children with cerebral palsy $\left(n^{\text {Subject }}=6, n^{\text {RUN }}=14\right)$ (b)

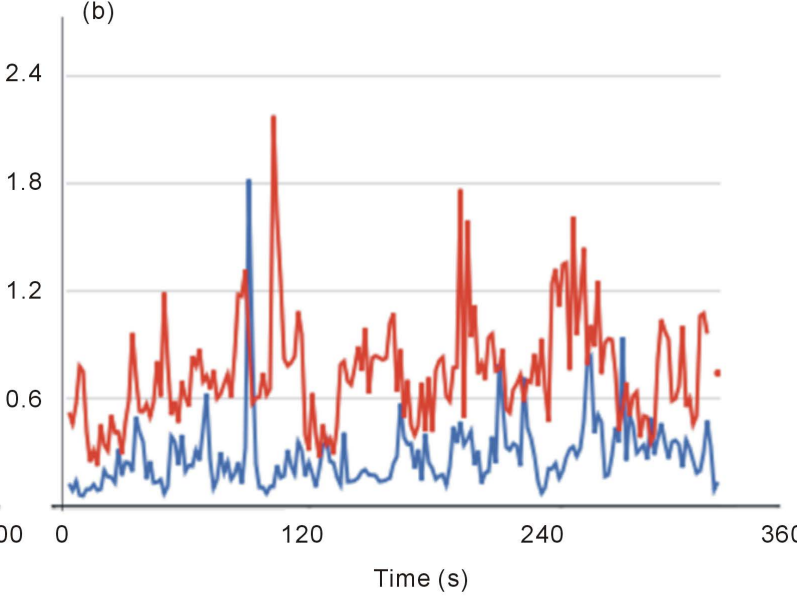

Typically developing (healthy) children $\left(n^{\text {subject }}=3, n^{\text {RUN }}=3\right.$ )

Children with cerebral palsy $\left(n^{\text {subject }}=6, n^{\text {RUN }}=13\right.$ )

Figure 2. Motor control ability during resting scan (a) real-time motion feedback used) and motor task scan ((b) real-time motion feedback was not used) collected from typically developing children group (blue) and CP group (red). (a) Both subject groups were able to keep their head still within the acceptable level $(M(t)<1 \mathrm{~mm})$. Towards the end of the scanning session $(t=298-300$ $\mathrm{sec}$ ), TDC subjects moved heads more than CP children. B. Both subject groups resulted in greater head motion when there was no real-time motion feedback provided. 
in relation to anatomical data and motor task data. Overlapping was found to occur in $74.28 \%$ (s.d. $=29.45 \%, p<0.05$ ) in ten left hemispheres and $74.83 \%$ (s.d. $=22.22 \%, p<0.05)$ in ten right hemispheres. The same region overlapped with the signal detected from the motor task scan by $93.59 \%$ (s.d. $=10.23 \%, p<$ 0.05 ) and $93 \%$ (s.d. $=12.29 \%, p<0.05$ ) in the same hemispheres, respectively, showing that anatomically derived ROI within the resting brain network is highly correlated to the functional data derived from the motor data (Figure 3). It was more challenging to identify the same regions in $\mathrm{CP}$ brains due to anatomical variances. However, as shown in Figure 3, the same strategy was used to identify the ROIs on the resting data, which were highly correlated with the motor data.

\subsection{Laterality Index as Measurement of Functional Connectivity}

Laterality index provided a metric that specified a predominant side for the cerebral activity in relation to the entire brain. A laterality index served as a measure of asymmetry between the two brain hemispheres. The results are shown in Figure 3. For the healthy subject and CP subjects, higher LI signifies that the subject's brain activity during the motor task was more lateralized to the left side or unaffected side, respectively. All three healthy subjects showed left hemisphere dominance while all, except one, CP subjects showed unaffected hemisphere dominance. This is consistent with the clinical data that is the healthy subjects' predominant right-sidedness while CP subjects' predominant preference for using unaffected side.

\section{Discussion}

Comparing activation differences before and after an intervention is a popular

(a)

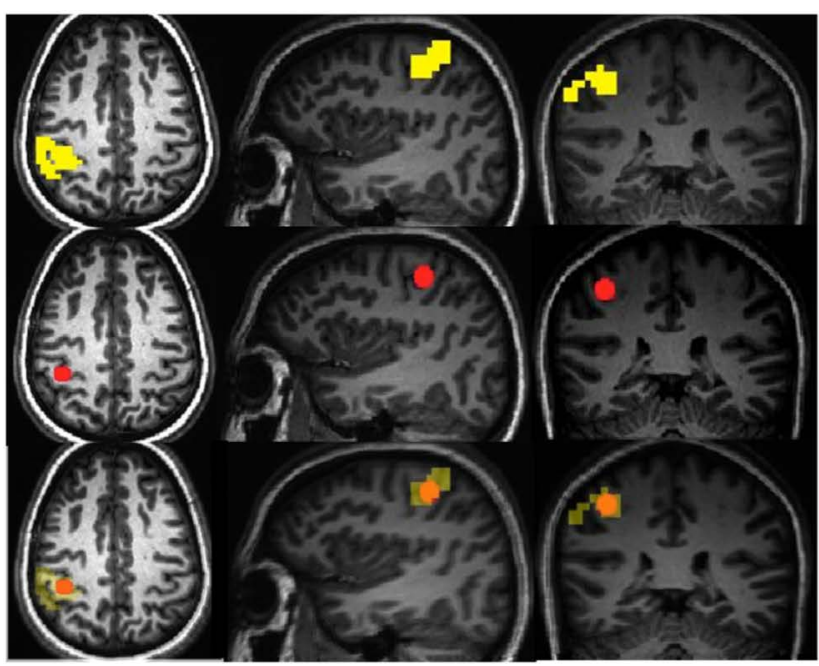

(b)

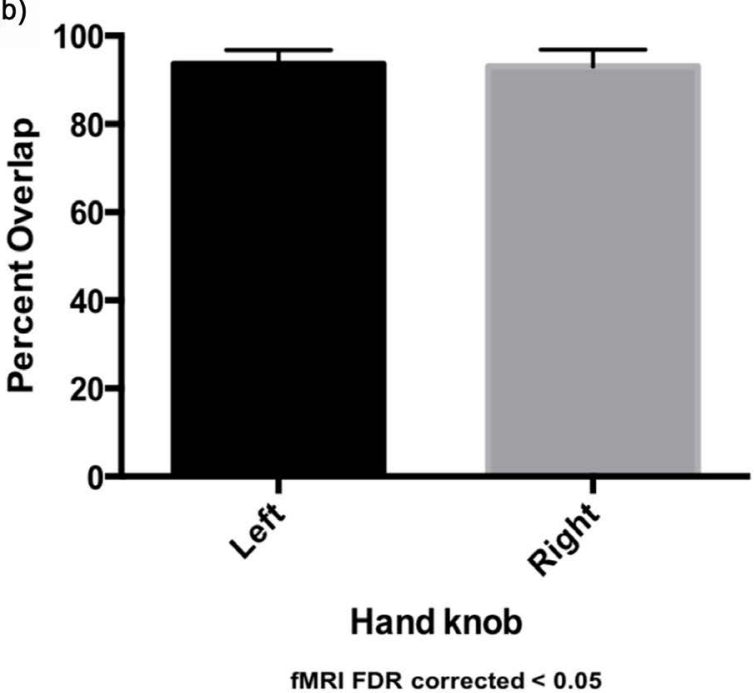

Figure 3. An example of demarcating a hand-knob region as a 6-mm sphere on a hemisphere, manually aligning the motor task data with this ROI as reference point, also showing an example of the resulted overlap between an ROI and corresponding motor signal (a). This alignment showed high overlap rate in both hemispheres (b). 
way to demonstrate a neuroplastic change presumed to occur as a result of an intervention [6] [7] [13], but is often unused because of questions surrounding the reliability subjects to complete scanning protocols in such a way that provides valid scanning data. Our goal was to implement a novel scanning methodology to help subjects with and without $\mathrm{CP}$, mainly using resting scan data. With the use of resting state scan, we aimed to perform seed-voxel analysis, which does not require heavy reliance on motor scan data, whose use is limited by motion artifacts, or whole-brain functional analysis, which is limited by anatomical variances.

In order to optimize the quality of resting-data scan, we incorporated real-time feedback system. The greatest benefit of this feedback system was demonstrated in Figure 2, where there was an appreciable difference between the subjects' ability to control the head motion during the resting scan. Another unexpected benefit was associated with the use of motion index as a parameter. This quantifiable parameter representing the amount of head motion allowed us to confirm that there was "salvageable" segments of data where children showed considerably little amount of head motion. Such salvageable data segments were found via identification of scanning segments with movement below acceptable thresholds. The duration of this salvageable segments corresponded to what has been previously suggested in the literature for the pediatric subjects' attention spans [14]. Another interesting result was that, after approximately 299 seconds, TDC (typically developing children) group showed increased MI, while CP children group showed decreased MI. Although CP showed greater levels of overall head and neck motions in comparison to children with typical development, this difference might be suggestive of different adaptive behaviors to real-time motion feedback between two groups.

Using the salvageable data, we were able to identify ROIs on the resting scan data, that was highly associated with the precentral "hand-knob" areas that are believed to be associated with hand movement [20] [17] [21] [22] [23] [24]. It has been studied by multiple researchers that these hand-knob areas showed correlation with the most active parts of the brain during the motor scans [25] [26] [27]; however it has not been widely studied the use of this hand-knob area for the resting state based analysis, such as seed-voxel analysis. According to our finding as shown in Figure 3, there were statistically significant correlations between these anatomical structures, our ROI on resting state data, and clusters of highly active voxels during the hand motion during the motor scan, implying that anatomical hand-knob areas can be reliably used for rest-state data analysis. Given that rest-state data analysis has been recognized as an effective way to study the default brain network [28] [29], which might hold more potential for investigating intrinsic neuroplasticity, being able to use this easily identifiable anatomical landmark for resting-state data analysis with certain level of confidence is promising.

In order to demonstrate its application, we proceed to perform a simple rest- 
ing-state analysis using these ROIs as "seeds" to derive interhemispheric laterality. In Figure 4, an example of laterality change within a CP subject's brain before and after a therapeutic intervention is introduced. This particular subject was a 10-year old male with quadriplegic cerebral palsy that caused him a fairly significant difficulty in controlling head motion, especially during the motor task. During both scans, the subject found it challenging to stay still in the scanner. His right motor control was better than his left motor control, but both were limited compared to typically developing or hemiplegic counterparts. However, he showed greatly enhanced laterality between two hemispheres as well as the overall strength of the brain activity during post-therapy scan, compared to pre-therapy scan. The implication is promising and suggestive of the positive effect potentially brought up with p-CIMT, which was the only variable between two scanning session.

Furthermore, Figure 5 demonstrates the use of laterality index (LI) as a quantifiable measure for the aforementioned inter-hemispheric laterality change. What is shown in this figure is based on the clinical data on 3 children with $\mathrm{CP}$ immediately to and after a CIMT program. It is noticeable that all children with $\mathrm{CP}$ who provided post-CIMT yielded LIs that were closer to zero, indicating establishment of neutrality after successfully completing CIMT. Six of the children with CP showed pre- and post-CIMT LI that were greater than 0 indicating that they have the cerebral networks that are lateralized in favor of using unaffected

(a)

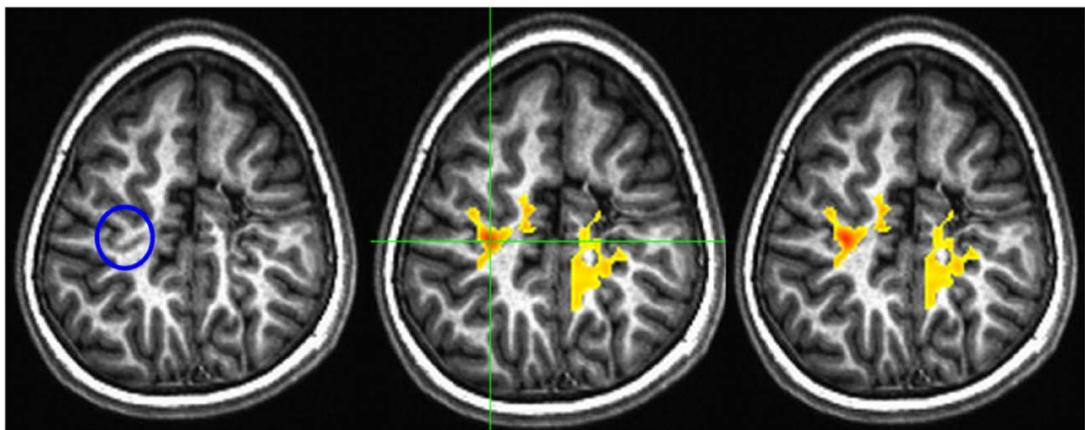

(b)

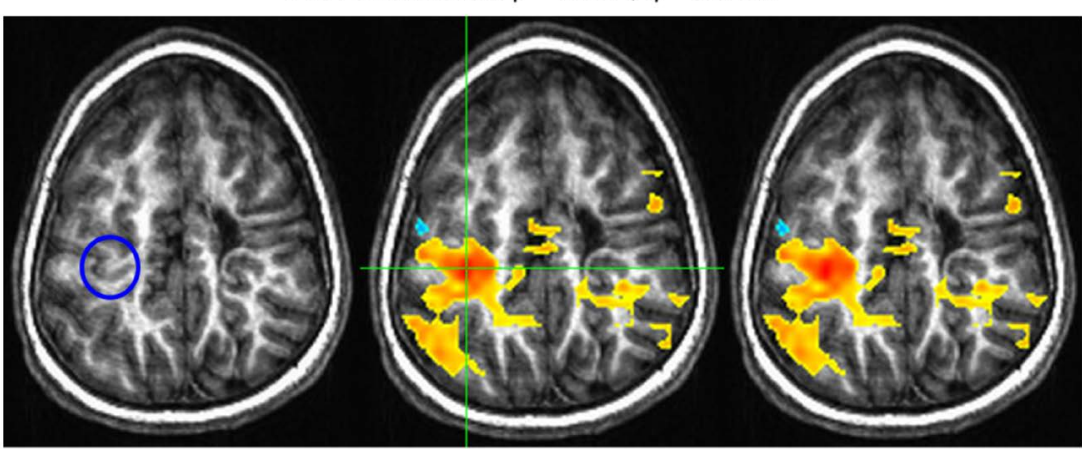

FDR Corrected at $p=0.01, q=0.0499$

Figure 4. Functional connectivity resulted from the protocol suggested in this study before (a) and after (b) the subject received p-CIMT. There is a difference between two lateralization patterns, potentially suggesting the effect of $\mathrm{p}$-CIMT. 
(a)

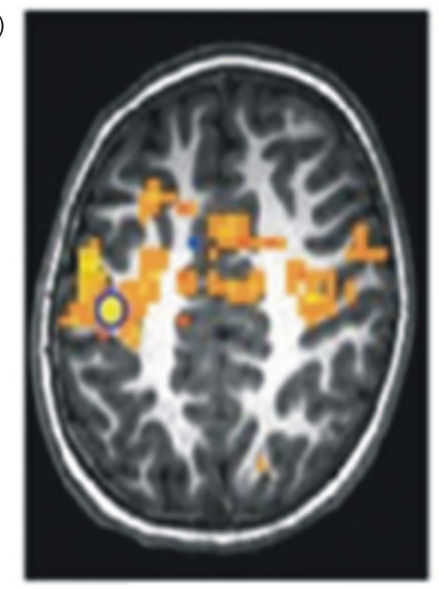

HEATHY CHILD (b)

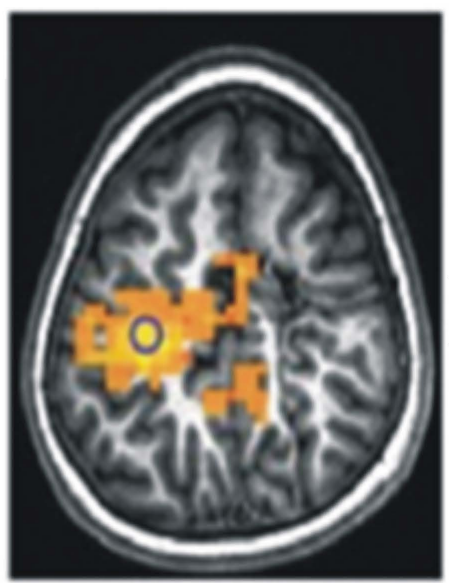

CHILD WITH CEREBRAL PALSY

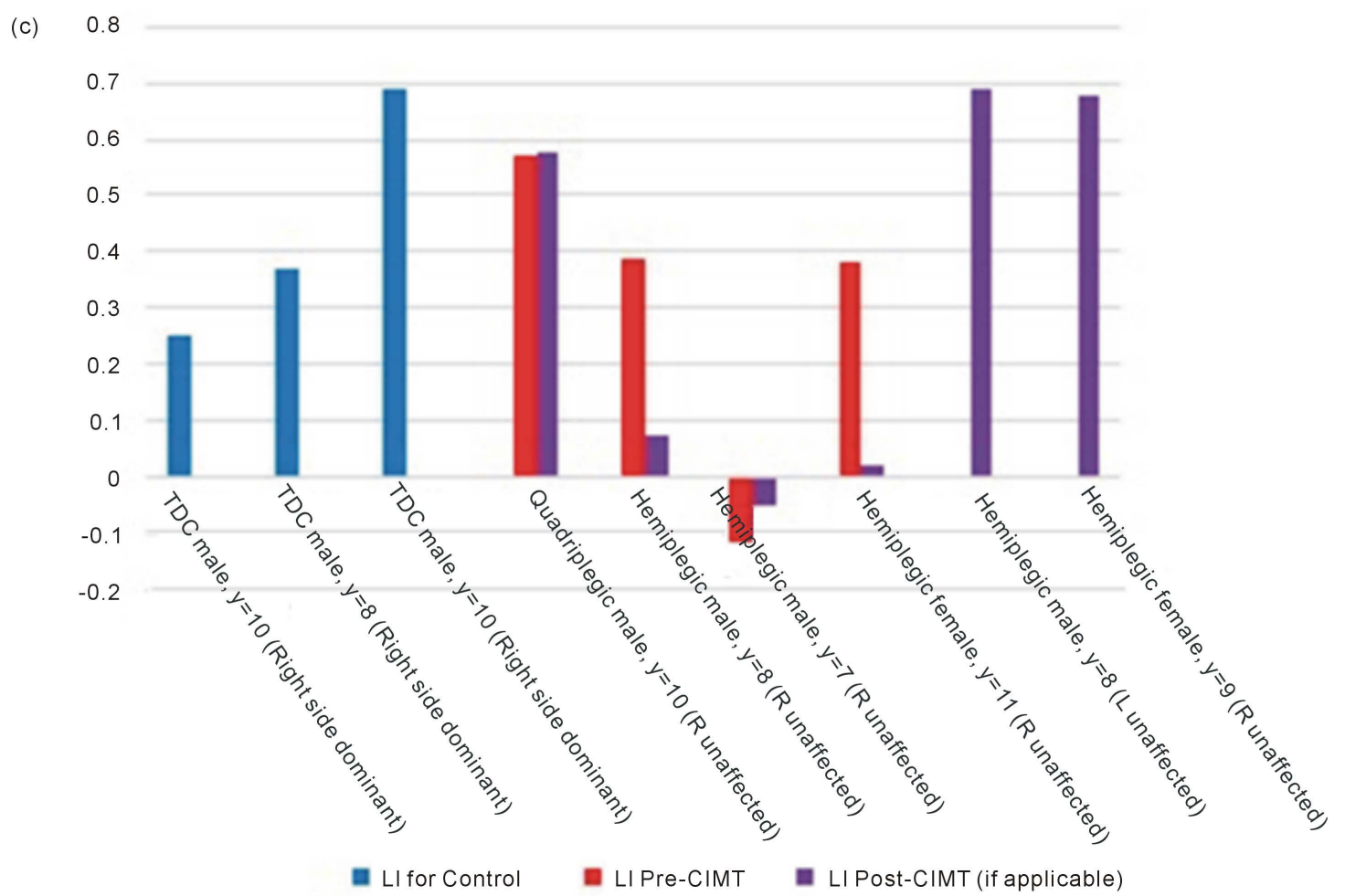

Figure 5. A set of false discovery rate (FDR) corrected examples of LI from a typically developing subject data (a) and CP subject data (b). For healthy subjects, $\mathrm{LI}=\mathrm{f}(\mathrm{QLH}-\mathrm{QRH}) /(\mathrm{QLH}+\mathrm{QRH})$ was used. For CP subjects, $\mathrm{LI}($ Unaffected $)=(\mathrm{U}-\mathrm{A}) /(\mathrm{U}+\mathrm{A})$ was used. Higher LI signifies that the subject's brain activity during the motor task was more lateralized to the left side or unaffected side, respectively. This is consistent with the clinical data that is the subject' predominant right-sidedness. (c) Calculated LI from a CP subject data represented in bar graph. LI for TDC are in blue marked as LI for control. Positive values are indicative of left hemisphere (LH) dominant in this case. Marked as red is LI for CP subjects before undergoing CIMT. Total four data sets were available. Marked as purple is LI for CP subjects after CIMT. All six subjects underwent post-therapy scan. Higher LI signifies that his brain activity during the motor task was more lateralized to the unaffected side of the brain.

side of the brain. One exception is shown in Figure 5 where the LI that is indicative of a preference for affected side of the brain, but the magnitude of the LI is much smaller compared to the other children. Considering that $\mathrm{CP}$ patients are 
subject to anatomical and physiological variances in neuronal networks, this exception may imply that there might be other variances that LI alone cannot measure.

In our study, we were able to demonstrate a methodology leading to a functional connectivity analysis for fMRI data obtained in children with parameters that are less dependent on motion effects. Our subsequent analysis methodology using seed ROIs succeeded in yielding functional connectivity data that is consistent with an expected clinical profile. Compared to the gold standard method for fMRI studies, which rely on motor scan data, instead of resting scan data, we were able to demonstrate that clinically accurate and meaningful data analyses can be carried on without being affected by anatomical variances or excessive head motion of the pediatric subjects. Analysis using this methodology to show functional connectivity of the brain before and after a therapy was also documented, but needs further investigation in order to be certain that clinical improvements can be associated with neural plastic changes in LI.

\section{Limitation and Future Directions}

The major limitation for this study is the small sample size. Also, automated determination of LI is challenging with gross anatomical structures inn children with specific diagnoses. Finally, investigators are considering another approach to optimize the results during motor task scanning via use of real-time motion feedback during this scanning period. This would create a confounding variable to the motor-task that is being utilized, but it is feasible that if real-time feedback were used during both resting state and motor-task scanning that patterns of activation could be apportioned correctly.

\section{References}

[1] Koman, L.A., Smith, B.P. and Shilt, J.S. (2004) Cerebral Palsy. Lancet, 363, 1619-1631. https://doi.org/10.1016/S0140-6736(04)16207-7

[2] Taub, E., Griffin, A., Uswatte, G., Gammons, K., Nick, J. and Law, C.R. (2011) Treatment of Congenital Hemiparesis with Pediatric Constraint-Induced Movement Therapy. Journal of Child Neurology, 26, 1163-1173. https://doi.org/10.1177/0883073811408423

[3] DeLuca, S.C., Ramey, S.L., Trucks, M.R. and Wallace, D.A. (2015) Multiple Treatments of Pediatric Constraint-Induced Movement Therapy (pCIMT): A Clinical Cohort Study. The American Journal of Occupational Therapy. Official Publication of the American Occupational Therapy Association, 69, Article ID: 6906180010. https://doi.org/10.5014/ajot.2015.019323

[4] DeLuca, S.C., Echols, K., Law, C.R. and Ramey, S.L. (2006) Intensive Pediatric Constraint-Induced Therapy for Children with Cerebral Palsy. Journal of Child Neurology, 21, 931-938. https://doi.org/10.1177/08830738060210110401

[5] Naidich, T.P., Valavanis, A.G. and Kubik, S. (1995) Anatomic Relationships along the Low-Middle Convexity: Part I-Normal Specimens and Magnetic Resonance Imaging. Neurosurgery, 36, 517-532.

https://doi.org/10.1227/00006123-199503000-00011 
[6] Reid, L.B., Boyd, R.N., Cunnington, R. and Rose, S.E. (2016) Interpreting Intervention Induced Neuroplasticity with fMRI: The Case for Multimodal Imaging Strategies. Neural Plasticity, 2016, Article ID: 2643491. https://doi.org/10.1155/2016/2643491

[7] Reid, L.B., Rose, S.E. and Boyd, R.N. (2015) Rehabilitation and Neuroplasticity in Children with Unilateral Cerebral Palsy. Nature Reviews. Neurology, 11, 390-400. https://doi.org/10.1038/nrneurol.2015.97

[8] Raschle, N., Zuk, J., Ortiz-Mantilla, S., Sliva, D.D., Franceschi, A., Grant, P.E. and Gaab, N. (2012) Pediatric Neuroimaging in Early Childhood and Infancy: Challenges and Practical Guidelines. Annals of the New York Academy of Sciences, 1252, 43-50. https://doi.org/10.1111/j.1749-6632.2012.06457.x

[9] Epstein, J.N., Casey, B.J., Tonev, S.T., Davidson, M., Reiss, A.L., Garrett, A. and Spicer, J. (2007) Assessment and Prevention of Head Motion during Imaging of Patients with Attention Deficit Hyperactivity Disorder. Psychiatry Research, 155, 75-82. https://doi.org/10.1016/j.pscychresns.2006.12.009

[10] Slifer, K.J., Cataldo, M.F., Cataldo, M.D., Llorente, A.M. and Gerson, A.C. (1993) Behavior Analysis of Motion Control for Pediatric Neuroimaging. Journal of Applied Behavior Analysis, 26, 469-470. https://doi.org/10.1901/jaba.1993.26-469

[11] Slifer, K.J., Koontz, K.L. and Cataldo, M.F. (2002) Operant-Contingency-Based Preparation of Children for Functional Magnetic Resonance Imaging. J App Beh Anal., 35, 191-194. https://doi.org/10.1901/jaba.2002.35-191

[12] Seghier, M.L. (2008) Laterality Index in Functional MRI: Methodological Issues. Magnetic Resonance Imaging, 26, 594-601.

https://doi.org/10.1016/j.mri.2007.10.010

[13] Bleyenheuft, Y., Dricot, L., Gilis, N., Kuo, H.-C., Grandin, C., Bleyenheuft, C. and Friel, K.M. (2015) Capturing Neuroplastic Changes after Bimanual Intensive Rehabilitation in Children with Unilateral Spastic Cerebral Palsy: A Combined DTI, TMS and fMRI Pilot Study. Research in Developmental Disabilities, 43-44, 136-149. https://doi.org/10.1016/j.ridd.2015.06.014

[14] Yuan, W., Altaye, M., Ret, J., Schmithorst, V., Byars, A.W., Plante, E. and Holland, S.K. (2009) Quantification of Head Motion in Children During Various fMRI Language Tasks. Human Brain Mapping, 30, 1481-1489. https://doi.org/10.1002/hbm.20616

[15] Yang, S., Ross, T.J., Zhang, Y., Stein, E.A. and Yang, Y. (2005) Head Motion Suppression Using Real-Time Feedback of Motion Information and Its Effects on Task Performance in fMRI. Neuroimage, 27, 153-162. https://doi.org/10.1016/j.neuroimage.2005.02.050

[16] Schwindack, C., Siminotto, E., Meyer, M., McNamara, A., Marshall, I., Wardlaw, J.M. and Whittle, I.R. (2005) Real-Time Functional Magnetic Resonance Imaging (rt-fMRI) in Patients with Brain Tumours: Preliminary Findings using Motor and Language Paradigms. British Journal of Neurosurgery, 19, 25-32. https://doi.org/10.1080/02688690500089621

[17] Yousry, T.A., Schmid, U.D., Alkadhi, H., Schmidt, D., Peraud, A., Buettner, A. and Winkler, P. (1997) Localization of the Motor Hand Area to a Knob on the Precentral Gyrus. A New Landmark. Brain: A Journal of Neurology, 120, 141-157. http://www.ncbi.nlm.nih.gov/pubmed/9055804 https://doi.org/10.1093/brain/120.1.141

[18] Rogers, B.P., Morgan, V.L., Newton, A.T. and Gore, J.C. (2007) Assessing Functional Connectivity in the Human Brain by FMRI. Magnetic Resonance Imaging, 
25, 1347-1357. https://doi.org/10.1016/j.mri.2007.03.007

[19] Pizzella, V., Tecchio, F., Romani, G.L. and Rossini, P.M. (1999) Functional Localization of the Sensory Hand Area with Respect to the Motor Central Gyrus Knob. Neuroreport, 10, 3809-3814. https://doi.org/10.1097/00001756-199912160-00016

[20] Sutcliffe, T.L., Gaetz, W.C. and Logan, W. (2007) Cortical Reorganization after Modified Contraint-Induced Movement Therapy in Pediatric Hemiplegic Cerebral Palsy. Journal of Child Neurology, 22, 1281-1287. https://doi.org/10.1177/0883073807307084

[21] Pantazis, D., Joshi, A., Jiang, J., Shattuck, D., Bernstein, L.E., Damasio, H. and Leahy, R.M. (2010) Comparison of Landmark-Based and Automatic Methods for Cortical Surface Registration. NeuroImage, 49, 2479. https://doi.org/10.1016/j.neuroimage.2009.09.027

[22] Garrison, K.A., Rogalsky, C., Sheng, T., Liu, B., Damasio, H., Winstein, C.J. and Aziz-Zadeh, L.S. (2015) Functional MRI Preprocessing in Lesioned Brains: Manual versus Automated Region of Interest Analysis. Frontiers in Neurology, 6, 196. https://doi.org/10.3389/fneur.2015.00196

[23] Boroojerdi, B., Foltys, H., Krings, T., Spetzger, U., Thron, A. and Topper, R. (1999) Localization of the Motor Hand Area using Transcranial Magnetic Stimulation and Functional Magnetic Resonance Imaging. Clinical Neurophysiology, 110, 699-704. https://doi.org/10.1016/S1388-2457(98)00027-3

[24] Toro, R., Fox, P.T. and Paus, T. (2008) Functional Coactivation Map of the Human Brain. Cerebral Cortex, 18, 2553-2559. https://doi.org/10.1093/cercor/bhn014

[25] Chen, G., Chen, G., Xie, C. and Li, S.-J. (2011) Negative Functional Connectivity and Its Dependence on the Shortest Path Length of Positive Network in the Resting-State Human Brain. Brain Connectivity, 1, 195-206. https://doi.org/10.1089/brain.2011.0025

[26] Tian, L.X., Jiang, T.Z., Wang, Y.F., Zang, Y.F., He, Y., Liang, M., Sui, M.Q., Cao, Q.J., Hu, S.Y. and Peng, M. (2006) Altered Resting-State Functional Connectivity Patterns of Anterior Cingulate Cortex in Adolescents with Attention Deficit Hyperactivity Disorder. Neuroscience Letters, 400, 39-43. https://doi.org/10.1016/j.neulet.2006.02.022

[27] Pawela, C.P., Biswal, B.B., Hudetz, A.G., Li, R., Jones, S.R., Cho, Y.R. and Hyde, J.S. (2010) Interhemispheric Neuroplasticity Following Limb Deafferentation Detected by Resting-State Functional Connectivity Magnetic Resonance Imaging (fcMRI) and Functional Magnetic Resonance Imaging (fMRI). NeuroImage, 49, 2467. https://doi.org/10.1016/j.neuroimage.2009.09.054

[28] Damaraju, E., Huang, Y.-M., Barrett, L.F. and Pessoa, L. (2009) Affective Learning Enhances Activity and Functional Connectivity in Early Visual Cortex. Neuropsychologia, 47, 2480-2487. https://doi.org/10.1016/j.neuropsychologia.2009.04.023

[29] Fan, Y., Wu, C., Liu, H., Lin, K., Wai, Y. and Chen, Y. (2015) Neuroplastic Changes in Resting-State Functional Connectivity after Stroke Rehabilitation. Frontiers in Human Neuroscience, 9, 546. https://doi.org/10.3389/fnhum.2015.00546 